\title{
Nonlinear scaling of foraging contacts with rodent population density
}

Benny Borremans ${ }^{1}$, Jonas Reijniers ${ }^{1,2}$, Nelika K. Hughes ${ }^{1,3}$, Stephanie S. Godfrey ${ }^{4}$,

Sophie Gryseels ${ }^{1}$, Rhodes H. Makundi ${ }^{5}$ and Herwig Leirs ${ }^{1}$

${ }^{1}$ Evolutionary Ecology Group, Univ. of Antwerp, Antwerp, Belgium

${ }^{2}$ Dept of Engineering Management, Univ. of Antwerp, Antwerp, Belgium

${ }^{3}$ School of BioSciences, Univ. of Melbourne, Melbourne, Australia

${ }^{4}$ School of Veterinary and Life Sciences, Murdoch Univ., Western Australia, Australia

${ }^{5}$ Pest Management Center, Sokoine Univ. of Agriculture, Morogoro, Tanzania

Corresponding author: B. Borremans, Evolutionary Ecology Group, Univ. of Antwerp,

Antwerp, Belgium. E-mail: bennyborremans@gmail.com

Decision date: $26-$ Sep-2016

This is the author manuscript accepted for publication and has undergone full peer review but has not been through the copyediting, typesetting, pagination and proofreading process, which may lead to differences between this version and the Version of Record.

Please cite this article as doi: [10.1111/oik.03623].

'This article is protected by copyright. All rights reserved.' 
(Abstract)

Density-dependent shifts in population processes like territoriality, reproduction, dispersal, and parasite transmission are driven by changes in contacts between individuals. Despite this, surprisingly little is known about how contacts change with density, and thus the mechanisms driving density-dependent processes. A simple linear contact-density function is often assumed, but this is not based on a sound basis of empirical data. We addressed this question using a replicated, semi-natural experiment in which we measured contacts at feeding stations between multimammate mice, Mastomys natalensis, across ten distinct, linearly increasing densities between 10 and 272 animals/ha. Unexpectedly, unique contacts increased not linearly but sigmoidally with density, which we attribute to the species' scramble competition mating system, small-scale dominance/avoidance and absence of territoriality. These results provide new insights into how species' characteristics can relate to density-dependent changes in contacts, and the unexpected shape of the contact-density function warrants that density-dependence in ecological models, such as parasite transmission models, must be parameterized with care. 


\section{Introduction}

Many ecological and behavioral processes in animal populations, such as dispersal, territoriality and disease transmission, are influenced by population density (Stilling 1988, Leirs et al. 1997, Coulson et al. 2001). The precise mechanisms driving density-dependent changes can be complex, difficult to quantify, and process-specific, but changes in density ultimately manifest themselves through behavioral interactions, and are perceived at the individual level as changes in the number of other individuals encountered (Ostfeld and Canham 1995, Krause et al. 2007, Sih et al. 2009). Information on how the properties of encounters (i.e. contacts) between individuals change with density would therefore provide a good basis for improving our understanding of the way in which population density drives ecological processes.

A process for which the importance of density-dependence of contacts is well established is parasite transmission (Anderson and May 1979). Parasites are often transmitted through direct contact between individuals, and the way in which contacts vary with density can determine whether or not an infection can persist in a population, as well as how fast it can spread (Anderson and May 1985, de Jong et al. 1995, Goyens et al. 2013). Yet, although accurate knowledge about density-dependence is necessary for understanding and modelling disease transmission, there are almost no cases in which the transmissiondensity or contact-density relationship has been described empirically, let alone quantified over a relevant range of densities. In their absence, infection models typically assume a linear correlation between density and contacts or transmission, and in some cases a more flexible power law (Smith et al. 2009, Hu et al. 2013), but rarely are these assumptions based on empirical data.

Changes in the perceived number of animals and the frequency and nature of contacts between individuals can affect processes linearly or nonlinearly. While dispersal is commonly assumed to be either positively or negatively density-dependent within species (Matthysen 2005), territorial and aggressive behavior often changes nonlinearly with density, where territoriality/aggression is primarily observed at intermediate densities because it is not necessary at low densities and too costly at high densities (Warner and Hoffman 1980, Maher and Lott 2000). In some processes, such as mating behavior, changes in the properties of contacts (e.g. increased contact frequency), can cause nonlinear behavioral shifts (Kokko and Rankin 2006, Adler 2011). For example, a monogamous mating system is more likely in species, or even populations within species, that generally occur at low population densities (Kleiman 1977), while polygamy and promiscuity are commonly observed at (seasonally) high densities (Endries and Adler 2005, Gerlach et al. 2012). Even within species, mating behavior can change depending on 
population densities (Emlen and Oring 1977, Travis et al. 1995). Echimyid rodents, for example, are generally more likely to be monogamous at low densities and polyandrous and/or polygynous (or potentially promiscuous) at higher densities (Adler 2011).

While changes in contacts with density are important for a wide range of ecological processes, contact structure is also relevant in itself. The way in which individuals are structured into a social network, how this network changes with density, and whether there is contact heterogeneity in the population will have consequences for the spread of information or disease in the population (Sih et al. 2009, Perkins et al. 2009). Some individuals may be more or less connected to others, and the way in which they are connected can influence many processes. For example, highly connected individuals and those that are central in their social network would be more likely to acquire and spread an infection than those that have few contacts (Anderson and May 1991, Lloyd-Smith et al. 2005). They may also receive more information about other individuals (Krause et al. 2007), or gain better access to mates for reproduction or mate guarding (Sih et al. 2009).

As contacts between individuals constitute an important proximate mechanism driving density-dependent changes in a wide range of population dynamic processes, much could be learned from quantifying the contact-density relationship, especially for species that experience regular density fluctuations. Measuring the effects of population density on contacts is difficult however, as it requires elaborate field studies or manipulative experiments. We know of only three species for which this has been done. In an enclosure experiment, male elk (Cervus canadensis) contact rates increased with density (unfortunately the shape of the contact-density could not be determined due to the limited range of densities) (Vander Wal et al. 2012) while, in a different study, contacts amongst females were found to increase in a nonlinear, convexup (power) fashion (Cross et al. 2013). This relationship was also observed for territorial field voles (Microtus agrestis) in a study in which contact networks were estimated using capture-mark-recapture data (Davis et al. 2015), and for brushtail possums (Trichosurus vulpecula) in a radio-telemetry study using trap locations of males inside the home ranges of females as a proxy for contacts (Ramsey et al. 2002). The few existing studies to date therefore suggest that a positive, convex-up power function may be common, but there are currently too few studies to draw any general conclusions about how contacts can be regulated by density.

We measured contacts in a semi-natural setting across a range of ten distinct population densities of the Natal multimammate mouse (Mastomys natalensis), a common African rodent known for its strongly fluctuating population densities (Leirs et al. 1997), and a known reservoir host for pathogens including Lassa virus (Frame et al. 1970). Multimammate mice have a promiscuous mating system where males are 
thought to use a scramble competition strategy for access to females (Kennis et al. 2008, Borremans et al. 2014). It is not territorial or aggressive (Veenstra 1958), home range overlap increases with density, and while home ranges of female adults increase in size with population density, those of subadults and male adults decrease (Borremans et al. 2014). We therefore predicted that contact rates and density would be positively correlated. As the species is not territorial and adapted to living at high densities, we further predicted that the correlation would be close to linear, although potentially with a density threshold at which contact rates increase less strongly due to decreasing home range sizes. Because the experiment was performed in the breeding season, we predicted that contacts between male and female adults would be higher than within sexes or with subadults. Finally, given that their environment is variable and individuals are not identical, we predicted that there would be significant heterogeneity in individual contact rates.

This study does not aim to determine the consequences of density-dependent changes in contacts for population dynamic processes. Rather, by establishing how contacts themselves change with density, we address a necessary step in the process of identifying the causes of density-dependence of ecological processes and the mechanistic way in which individual behavior drives these processes.

\section{Materials and methods}

\section{Animals and enclosures}

In May 2012, 352 M. natalensis individuals were trapped in fallow fields (4 fields of 1 ha spaced at least $1 \mathrm{~km}$ apart) on the campus of the Sokoine University of Agriculture (SUA) in Morogoro (Tanzania) and transported to the animal house of the SUA Pest Management Centre. There, groups of four same-sex individuals were housed together in cages $(28 \times 11.5 \times 12 \mathrm{~cm}$, food and water ad libitum $)$ until the start of the experiment, maximum 1 week after being captured. A Radio Frequency Identification (RFID) Passive Integrated Transponder (PIT) tag of which the unique ID can be detected using an RFID antenna (Francis Scientific Instruments, Bluntisham, Cambridge, UK) was implanted subcutaneously in each animal. Sex and reproductive status (adult/subadult following (Leirs 1994)) were recorded before the start of the experiment.

The experiment was conducted in four 0.5 ha $(70 \times 70 \mathrm{~m})$ enclosed fields on the SUA campus. The habitat in the fields was the same as that in the surrounding fallow fields where the experimental animals were captured, consisting of a mix of grasses and shrubs, providing natural cover against predation and weather. Mouse-proof enclosures were constructed of galvanized steel, $110 \mathrm{~cm}$ above and $70 \mathrm{~cm}$ below ground in a gravel-filled ditch. As these enclosures were almost 10 times larger than the mean $M$. 
natalensis home range (Borremans et al. 2014), spatial behavior was unlikely to be affected by the experimental setup.

The four enclosures were randomly assigned to a low (LD) or high (HD) population density treatment, where the LD enclosures (A and B) contained 5 to 40 animals, and HD enclosures (C and D) contained 60 to 136 animals over the course of the experiment (Table 1). This constitutes a range of densities representative of natural fluctuations in the study area (Leirs 1994). New animals were added to the enclosures every 7 days, adding to those already present (Table 1).

Individuals that were housed in the same cage after trapping were randomly assigned to different enclosures, ensuring that animals in an enclosure were unfamiliar with each other unless in the rare event they had occurred together in the field before capture. Individual release locations within an enclosure were chosen randomly, stratified by enclosure quadrant to avoid introducing clustering. After release at the beginning of each treatment week, animals were given two days to acclimatize before recording began (i.e. each treatment week consisted of 2 adaptation days followed by 5 recording days). The experimental period coincided with the beginning of the annual breeding season, during which densities are usually low (10-30/ha). As a result, the animals were exposed to densities they would not normally encounter during this period, but unfortunately this is an unavoidable artefact of this kind of field experiment.

All field work was conducted in compliance with the laws, regulations and policies on humane care and handling of animals for research purposes guided by the 'Code of Conduct for Research Ethics of Sokoine University of Agriculture, Tanzania' (Revised 2012) and Tanzania's Animal Welfare Act of 2008.

We assumed mortality rates during the experiment (e.g. from avian predators) to be zero, as there was a highly significant linear correlation (Pearson's product moment correlation test; $t=10.5, d f=18, R^{2}=$ $0.86, P<0.0001)$ between the experimental densities and the number of recorded animals.

We tested whether the addition of new animals to the existing populations affected contacts, but the number of unique contacts was not different between newly and previously added animals (ANOVA of a linear mixed model with individual as random effect; addition status (new/previous) effect estimate $\left.=-0.04 \pm 0.06, \quad \chi_{1}^{2}=0.41, \mathrm{P}=0.52\right)$. We therefore did not take into account an addition effect.

\section{Feeding stations and visitation recording}

Each enclosure contained nine feeding stations consisting of a plastic tube/food-container $(10 \mathrm{~cm}$ diameter, $20 \mathrm{~cm}$ length) placed horizontally in a wire mesh tube $(18 \mathrm{~cm}$ diameter, $45 \mathrm{~cm}$ length) that had 
only one entry. Feeding stations were replenished daily with a mixture of peanut butter, maize flour and seeds. Four feeding stations (one in the center of each quadrant) were equipped with an RFID antenna at the entry to record the passage time of each RFID tag-equipped mouse. The five remaining feeding stations were placed evenly in the enclosure in order not to artificially attract all animals to the four antenna-equipped stations (see SI Figure S1 for a layout sketch). This arrangement ensured that at least one feeding station was present in the average home range $\left(600 \mathrm{~m}^{2}\right)$ of an animal (Borremans et al. 2014), minimizing the influence of station placement on natural spatial behavior. As $M$. natalensis is an opportunistic, generalist forager (Leirs et al. 1994, Odhiambo et al. 2008, Mulungu et al. 2011), the situation in which multiple individuals are attracted to a food source (e.g. local presence of fruit, seeds or nuts, a large arthropod, a recently dead animal, etc.) could easily occur in a natural setting. While the use of feeding stations limits our study to the measurement of foraging-related contacts, they are likely to be representative for the average contact, as foraging is likely to be their main activity, and assuming that other related behavioral traits such as social hierarchy or mating behavior do not entirely disappear when foraging.

Because multimammate mice are strictly nocturnal, recording was done from $6 \mathrm{pm}$ until 7 am. Due to a technical problem, one of the four antennas in an enclosure did not function properly for a number of days, resulting in some missing data (see SI Section 2 for details). We assessed the possible effect of a missing antenna using simulations, and found that this did not significantly change mean degree patterns (SI Table S1), which means that we could safely compare all data.

\section{Estimating contacts}

Two individuals were considered to have had a contact if they visited the same feeding station within a certain time interval (e.g. Clay et al. 2009). To make an informed choice, the effect of time interval size on contact patterns was examined by calculating contacts for a range of different intervals (1-300 s). This did not result in qualitative differences in contact patterns (SI Figures S3 and S4), so we chose a recording interval of 30 s because it is sufficiently short to be a meaningful approximation of a contact yet long enough to provide sufficient data. We constructed unweighted, undirected networks for each antenna in each enclosure and in each observation period, where each edge represented whether a pair of individuals contacted each other within the observation period (5 days). An unweighted (as opposed to weighted) network was chosen because the weight of each edge was directly dependent on the arbitrary choice of contact time, and because we are mainly interested in how the mean number of unique contacts changes with population density, which does not require the use of weighted contacts. By measuring 
contacts, we can make inferences about the transfer of information and directly transmitted (through physical or indirect environmental contact) diseases.

\section{Connectivity}

Connectivity was investigated using a number of standard social network measures relating to different aspects of the contact structure (Croft et al. 2007, Krause et al. 2007, Whitehead 2008). Because most animals (193/218) had contacts in only a single antenna, each antenna was treated as a separate, statistically independent social network. This resulted in 80 networks (four antennas $\mathbb{x}$ two enclosures $\mathbf{x}$ ten observation weeks). For each antenna, we calculated mean degree, mean betweenness and mean closeness (Croft et al. 2007, Whitehead 2008).

Each individual's degree (Sih et al. 2009) was calculated as the number of other individuals it had contacted during a recording week, regardless of the number of times this connection was recorded (i.e. unweighted degree). Degree heterogeneity was calculated for the highest density treatment $(\mathrm{N}=136$, data from two enclosures merged), for maximum sample size. To assess the biological significance of the observed degree patterns, mean degree was compared with that of random networks. For each enclosure and density treatment, network randomization was done by randomly re-assigning an antenna location to an individual for a given observation period, and re-calculating the networks (1,000 iterations). This permutation method ensured that all behavioral and environmental factors (e.g. home range, activity time, number of visits) were retained, while randomizing contacts. The P-value was given by the proportion of permuted networks for which mean degree was larger than the observed value.

Betweenness is the number of shortest indirect connections between any two individuals in the network that pass through a focus individual, and is a measure of how central an individual is in a network (Freeman 1977). Individuals with high betweenness do not necessarily have many contacts, but rather function as important connections between subgroups in the network. These individuals would be more likely to acquire an infection or receive information, and can play a key role in controlling the flow of information or disease in a social network (Sih et al. 2009, Perkins et al. 2009). Betweenness is defined as

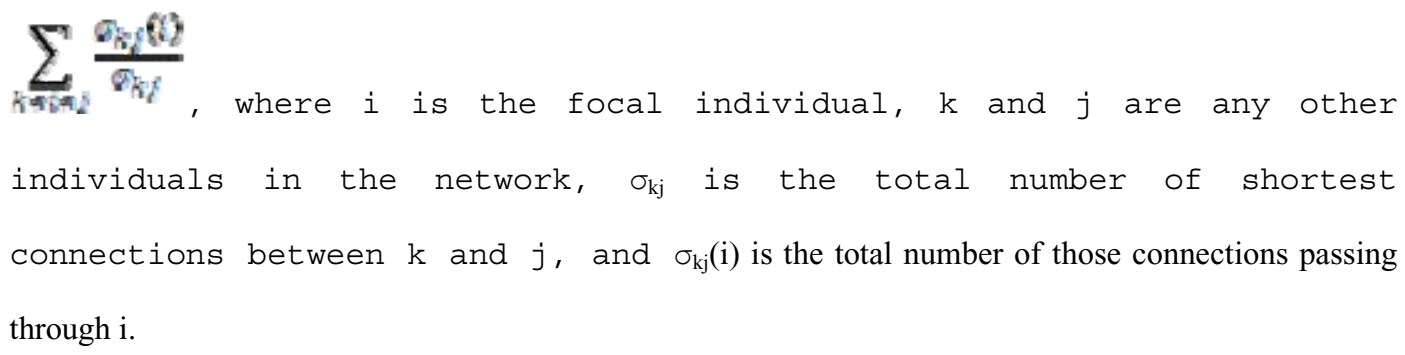


Closeness, defined as $\sum_{j}^{1}$ measure of how connected an individual is to others in the network (Beauchamp 1965, Opsahl et al. 2010). A closeness value of 0 occurs when an individual is unconnected to others, while higher closeness values indicate that the average distance of an individual to all other individuals is small. Individuals with high closeness would be more likely to acquire and transmit social information or an infection ("super spreaders") in a population (Corner et al. 2003), and may be more dominant in a hierarchic social organization (Sih et al. 2009).

We tested whether the network means of the different parameters (degree, betweenness, closeness) correlate significantly with density, using ANOVA of a linear mixed model with the (log-transformed) mean values as dependent variable, population density as fixed effect and enclosure as random effect.

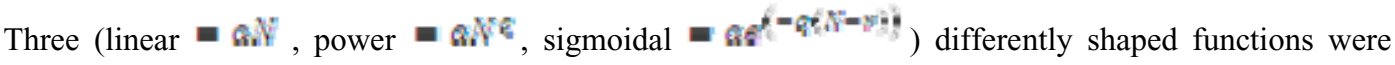
fitted to each parameter-density correlation using maximum likelihood, assessing model fit through AIC and comparing the different model fits using likelihood ratio tests.

A Mann-Whitney $U$ test (1,000 permutations) using a normalized $u$ value, where for each network the original data are compared to randomized data where the node attribute of interest is permuted (Croft et al. 2007), was used to test for effects of sex and reproductive age (juvenile/adult) on degree, betweenness and closeness.

\section{Frequency distribution of degree, betweenness and closeness}

In order to investigate the relative distribution of degree, betweenness and closeness within density treatments, these parameters were normalized for each density, with normalized degree $\mathrm{D}(i)_{\mathrm{norm}}=\frac{D(\mathrm{~S})}{n-1}$,

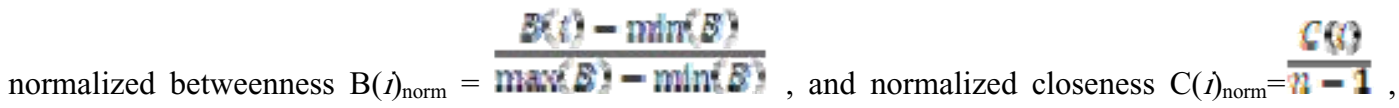
where $i$ is the focal individual and $n$ is the number of individuals in the network. In order to test whether the relative distribution of connectivity changes with population density, we calculated skewness of the normalized values within each density treatment, and tested (ANOVA) for a linear relationship between density and skewness. Positive or negative skewness values indicate a right-tailed or left-tailed frequency distribution, respectively.

Individual repeatability of normalized degree was estimated using a binomial generalized linear mixed model with logit link function, assuming an additive overdispersion parameter and a likelihood ratio test 
for significance, as described in (Nakagawa and Schielzeth 2010), using R package rptR (Stoffel et al. 2011).

Because in disease transmission a "20/80 rule" has been observed for a number of human diseases, where $20 \%$ of individuals was found to be responsible for $80 \%$ of transmission events (Woolhouse 1997), we calculated the proportion of individuals that was responsible for $80 \%$ of contacts.

\section{Activity time}

We investigated the times when an individual was active, and whether they changed with population density. For each individual, the number of minutes in which it was recorded was calculated for each density. More specifically, if an individual was recorded within the space of a certain minute, whether it was once or several times, this minute was counted as an active minute. Next, as a measure of the spread of an individual's activity time, we calculated the coefficient of variation of activity time for each individual within each density. We tested whether the number of active minutes (log-transformed) and the coefficient of variation increased significantly with density using ANOVA of a linear mixed model with density as fixed effect and individual as random effect. Additionally, because no studies have so far quantified the general activity pattern of $M$. natalensis at the population level, we calculated the number of recorded individuals within each 10-minute block. For maximum sample size this was done for the highest density treatment.

Data preparation, analyses and plotting were done in $\mathrm{R}$ ( $\mathrm{R}$ Core Team 2014) using packages plotrix (Lemon 2006), lme4 (Bates et al. 2014), sna (Butts 2014), network (Butts et al. 2014) and moments (Komsta and Novomestky 2015).

\section{Results}

\section{Connectivity}

All contact networks are shown in SI Figures S5-S14. Mean degree increased significantly with population density $(F=37.57, d f=18, P<0.0001$; Figure 1$)$, with a sigmoidal function resulting in the

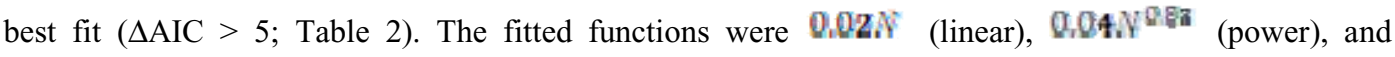
$\left(\frac{2.13}{(1+6)(-010(7-8049)}\right)$

(sigmoid). Contacts were observed even at the lowest densities $(\mathrm{N}=5)$, increased around $\mathrm{N}=40$, and reached a plateau at $\mathrm{N}=80$ (Figure 1). There was heterogeneity in degree, where $38 \%$ of individuals were responsible for $80 \%$ of connections, and $56 \%$ of all connections could be attributed to $20 \%$ of individuals. For 11 of the 20 (one for each enclosure-density combination) networks, 
mean degree was significantly larger than that in permuted networks, while the remaining networks showed the same trend (Figure 2).

Mean closeness and betweenness both increased significantly with density (Figure 3; Closeness: $F=$ 10.19, $d f=10, P=0.009$; Betweenness: $F=23.64, d f=12, P=0.0004)$, with no significant difference between the fit of differently shaped functions (Table 2). Neither degree, betweenness nor closeness correlated significantly with sex or reproductive age (Mann-Whitney $u$ test, $P>0.05$ for all networks at all densities).

\section{Frequency distribution of degree, betweenness and closeness}

Normalized degree was significantly different between individuals $\left(\chi^{2}{ }_{1}=26.5, P<0.0001\right)$, albeit with a low repeatability value (intra-class correlation coefficient) of $0.076 \pm 0.001$. The frequency distributions of normalized degree and betweenness (Figure 4) were both right-tailed and highly skewed (mean across densities $1.1 \pm 0.2$ and $1.4 \pm 0.4$, respectively), but skewness did not correlate significantly with population density for either degree $(F=0.73, d f=8, P=0.42)$ or betweenness $(F=0.41, d f=8, P=0.54)$.

The frequency distribution of normalized closeness was highly variable, with a mean skewness across densities of $0.15 \pm 0.2$ that ranged from -0.7 to 1.4 (Figure 4 ). There was no significant correlation between normalized closeness and population density $(F=0.02, d f=8, P=0.91)$.

\section{Activity time}

At the population level, there was a clear bimodal activity pattern, with peaks shortly after dusk (7pm) and at dawn (6am) (SI Figures S15). Although there was a trend towards an increase in the number of individual active minutes with population density, this was not significant when taking into account individual effects $\left(\chi^{2}{ }_{1}=3.396, P=0.065\right.$, Figure 5), but it was supported by a highly significant increase in the individual coefficient of variation of the time of activity with population density $\left(\chi^{2}{ }_{1}=26.42, d f=\right.$ $1, P<0.0001$, Figure 5).

\section{Discussion}

For a non-territorial, promiscuous species such as $M$. natalensis that regularly experiences high population densities, we predicted a positive correlation between density and degree. While we indeed found evidence for a positive effect of density, this was only observed at intermediate densities. At low and high densities, degree remained stable despite changes in density, an unexpected, sigmoid pattern that may have important consequences. 
At the lowest densities degree was relatively high compared to random data, which suggests that mice increase their efforts to find others and maintain contacts. This could be explained by the breeding behavior of $M$. natalensis, a promiscuous species, whose breeding season coincides with low densities. During this period, sexually active males are assumed to utilize a scramble competition mating strategy where they roam around to find as many receptive females as possible (Kennis et al. 2008, Borremans et al. 2014), a behavior that could indeed encourage the maintenance of contacts at low densities. This also implies that an Allee effect, where local extinction of the population becomes inversely densitydependent at low densities due to the breakdown of social structure (Allee 1931, Courchamp et al. 1999), would be unlikely. Degree levels observed at slightly higher densities $(\mathrm{N}=20,30)$ were similar to those at the lowest densities. They were situated within the range of those of random data, which may indicate that increased efforts for maintaining contacts were not necessary at these densities.

The increase in degree at intermediate densities likely results from an increase in home range overlap. A positive correlation between home range overlap and density has indeed been observed for this species (Borremans et al. 2014), and this would logically be expected to correspond to an increase in the number of individuals encountering each other. This increase is finite however, and appears to plateau from approximately 160 mice/ha. The most likely explanation for this would be avoidance at high densities, which is here supported by changes in the activity patterns, where at higher densities individuals are observed more often and at a wider range of observation times. This suggests that foraging visits become shorter but more frequent, possibly because of increased competition for resources. This finding supports the idea that $M$. natalensis exhibits non-aggressive avoidance behaviour in combination with scramble mate competition, and fits with the observation that home ranges of subadults and male adults decrease with population density (Borremans et al. 2014). A consequence of this wider range of contacts is that betweenness and closeness both increase, which would facilitate the flow of information or an infection through the social network

At the individual level, we observed strong individual heterogeneity in degree. Considering that there was significant individual repeatability in degree, it seems that individuals inherently differ in the number of contacts they have, but this was not related to sex or reproductive age and could still vary strongly as indicated by the low repeatability value. The existence of high-contact individuals is likely to be important for connectivity, and can have implications for processes like disease transmission (Woolhouse 1997, Lloyd-Smith et al. 2005), information transfer (Sih et al. 2009) or the evolution of behavioral strategies (Krause et al. 2007). 
Nonlinear, sigmoidal scaling of unique contacts with density in M. natalensis therefore seems to correspond with non-territoriality, promiscuous mating and scramble competition for mates, and smallscale avoidance at high densities. It is likely that parasite transmission is affected by this sigmoidal pattern, particularly the relatively high degree at low densities, which may ensure persistent parasite transmission during low-density periods. Very little is known however about how exactly a sigmoid contact-density function would affect the transmission of infections compared to the classic linear functions, and further in-depth modelling or experimental studies are highly needed.

The patterns observed and discussed here are likely determined by the specific ecological and behavioral traits of M. natalensis, and although they may represent general patterns, more comparable studies on species with different ecological and behavioral traits are needed before generalizations can be made. Evidence for a positive effect of density on contact rates has also been observed for field voles (Microtus agrestis), male brushtail possums (Trichosurus vulpecula), elk (Cervus canadensis) with the latter adapted to living in groups that frequently change in composition (Ramsey et al. 2002, Vander Wal et al. 2012, Cross et al. 2013, Davis et al. 2015). Furthermore, male brushtail possums seem to increase their home ranges at lower densities, possibly in order to maintain contacts with females (Ramsey et al. 2002), which corresponds to our observations for $M$. natalensis, suggesting that, in general, mate searching behavior can result in the maintenance of high contact rates even at low densities. In species that are territorial only at intermediate densities (Maher and Lott 2000), we predict that the number of unique contacts should increase at low densities but remain stable at intermediate densities due to the manifestation of territorial behavior, after which they can strongly increase when crossing a threshold density at which territoriality disappears. An enclosure experiment in which densities were manipulated found that movement distances significantly decreased with density in territorial meadow voles (Microtus pennsylvanicus), which may indeed correspond with lower contact rates (Ostfeld and Canham 1995). On the other hand, a positive, nonlinear (convex-up) relationship was observed for field voles (M. agrestis) (Davis et al. 2015), suggesting that it may not be straightforward to unravel the effect of density on different contact-related behavioral mechanisms.

\section{Conclusion}

In this study we empirically and quantitatively estimated the relationship between population density and contacts, and relate this to ecological and behavioral species characteristics. Before generalizations can be made, however, a larger context of model systems is needed, where we especially need data on species that are territorial, live at lower densities or experience irregular population fluctuations. Importantly, our 
results warn against assuming linear over nonlinear scaling of contacts with density, particularly in the case of parasite transmission.

\section{Acknowledgements}

Thanks to the excellent field work by the staff at the Pest Management Center (Sokoine University of Agriculture), in particular Shabani Lutea and Geofrey Sabuni. Thanks to Arne Iserbyt and Luc De Bruyn for help in the field. This work was supported by the University of Antwerp grant number GOA BOF FFB3567, Deutsche Forschungsgemeinschaft Focus Program 1596 and the Antwerp Study Centre for

Infectious Diseases (ASCID). Benny Borremans was a PhD fellow of Research Foundation Flanders (FWO) during the experiment. We have no competing interests. 


\section{References}

Adler, G. H. 2011. Spacing patterns and social mating systems of echimyid rodents. - J. Mammal. 92: 3138.

Allee, W. 1931. Animal aggregations: A study in general sociology. - The University of Chicago Press.

Anderson, R. M. and May, R. M. 1979. Population biology of infectious diseases: Part I. - Nature 280: $361-367$.

Anderson, R. M. and May, R. M. 1985. Vaccination and herd immunity to infectious diseases. - Nature 318: $323-329$.

Anderson, R. M. and May, R. M. 1991. Infectious Diseases of Humans: Dynamics and Control. - Oxford University Press.

Bates, D. et al. 2014. lme4: Linear mixed-effects models using Eigen and S4. R package version 1.1-7, http://CRAN.Rproject.org/package=lme4. in press.

Beauchamp, M. 1965. An improved index of centrality. - Behav. Sci. 10: 161-163.

Borremans, B. et al. 2014. Happily together forever: temporal variation in spatial patterns and complete lack of territoriality in a promiscuous rodent. - Popul. Ecol. 56: 109-118.

Butts, C. 2014. sna: Tools for social network analysis. R package version 2.3-2. in press.

Butts, C. et al. 2014. network: Classes for Relational Data. R package version 1.11.3. in press.

Clay, C. A. et al. 2009. Contact heterogeneity in deer mice: implications for Sin Nombre virus transmission. - Proc. R. Soc. B Biol. Sci. 276: 1305-1312.

Corner, L. A. L. et al. 2003. Social-network analysis of Mycobacterium bovis transmission among captive brushtail possums (Trichosurus vulpecula). - Prev. Vet. Med. 59: 147-167.

Coulson, T. et al. 2001. Age, sex, density, winter weather, and population crashes in Soay sheep. Science 292: 1528-1531.

Courchamp, F. et al. 1999. Inverse density dependence and the Allee effect. - Trends Ecol. Evol. 14: 405410.

Croft, D. et al. 2007. Exploring animal social networks. - Princeton University Press.

Cross, P. et al. 2013. Female elk contacts are neither frequency nor density dependent. - Ecology 94 : 2076-2086.

Davis, S. et al. 2015. Spatial analyses of wildlife contact networks. - J. R. Soc. Interface 12: 20141004.

de Jong, M. C. M. et al. 1995. How does transmission of infection depend on population size? - Publ. Newt. Inst. 5: 84-94.

Emlen, S. and Oring, L. 1977. Ecology, sexual selection, and the evolution of mating systems. - Science 197: 215-223.

Endries, M. J. and Adler, G. H. 2005. Spacing patterns of a tropical forest rodent, the spiny rat (Proechimys semispinosus), in Panama. - J. Zool. 265: 147-155.

Frame, J. D. et al. 1970. Lassa fever, a new virus disease of man from West Africa. 1. Clinical description and pathological findings. - Am. J. Trop. Med. Hyg. 19: 670-676.

Freeman, L. 1977. A set of measures of centrality based upon betweenness. - Sociometry 40: 34-41.

Gerlach, N. M. et al. 2012. Promiscuous mating produces offspring with higher lifetime fitness. - Proc. R. Soc. B Biol. Sci. 279: 860-866.

'This article is protected by copyright. All rights reserved.' 
Goyens, J. et al. 2013. Density thresholds for Mopeia virus invasion and persistence in its host Mastomys natalensis. - J. Theor. Biol. 317: 55-61.

$\mathrm{Hu}, \mathrm{H}$. et al. 2013. The scaling of contact rates with population density for the infectious disease models. - Math. Biosci. 244: 125-134.

Kennis, J. et al. 2008. Polyandry and polygyny in an African rodent pest species, Mastomys natalensis. Mammalia 72: 150-160.

Kleiman, D. 1977. Monogamy in mammals. - Q. Rev. Biol. 52: 39-69.

Kokko, H. and Rankin, D. J. 2006. Lonely hearts or sex in the city? Density-dependent effects in mating systems. - Philos. Trans. R. Soc. B Biol. Sci. 361: 319-334.

Komsta, L. and Novomestky, F. 2015. moments: Moments, cumulants, skewness, kurtosis and related tests. in press.

Krause, J. et al. 2007. Social network theory in the behavioural sciences: potential applications. - Behav. Ecol. Sociobiol. 62: 15-27.

Leirs, H. 1994. Population ecology of Mastomys natalensis (Smith, 1834). Implications for rodent control in Africa. - Belgian Administration for Development Cooperation, Agricultural Ed. Nr35, Brussels.

Leirs, H. et al. 1994. The basis of reproductive seasonality in Mastomys rats (Rodentia: Muridae) in Tanzania. - J. Trop. Ecol. 10: 55-66.

Leirs, H. et al. 1997. Stochastic seasonality and nonlinear density-dependent factors regulate population size in an African rodent. - Nature 389: 176-180.

Lemon, J. 2006. Plotrix: a package in the red light district of R. - R News 6: 8-12.

Lloyd-Smith, J. O. et al. 2005. Superspreading and the effect of individual variation on disease emergence. - Nature 438: 355-359.

Maher, C. R. and Lott, D. F. 2000. A review of ecological determinants of territoriality within vertebrate species. - Am. Midl. Nat. 143: 1-29.

Matthysen, E. 2005. Density-dependent dispersal in birds and mammals. - Ecography (Cop.). 28: 403416.

Mulungu, L. S. et al. 2011. Dietary differences of the multimammate mouse, Mastomys natalensis (Smith, 1834), across different habitats and seasons in Tanzania and Swaziland. - Wildl. Res. 38: 640-646.

Nakagawa, S. and Schielzeth, H. 2010. Repeatability for Gaussian and non-Gaussian data: a practical guide for biologists. - Biol. Rev. Camb. Philos. Soc. 85: 935-956.

Odhiambo, R. et al. 2008. Dietary selection in Mastomys natalensis (Rodentia: Muridae) in the maize agro-ecosystems of central and southwestern Tanzania. - Mammalia 72: 169-177.

Opsahl, T. et al. 2010. Node centrality in weighted networks: generalizing degree and shortest paths. Soc. Networks 32: 245-251.

Ostfeld, R. S. and Canham, C. D. 1995. Density-dependent processes in meadow voles: an experimental approach. - Ecology 76: 521-532.

Perkins, S. E. et al. 2009. Comparison of social networks derived from ecological data: implications for inferring infectious disease dynamics. - J. Anim. Ecol. 78: 1015-1022.

R Core Team 2014. R: A language and environment for statistical computing. in press.

Ramsey, D. et al. 2002. The effects of reducing population density on contact rates between brushtail possums: implications for transmission of bovine tuberculosis. - J. Appl. Ecol. 39: 806-818. 
Sih, A. et al. 2009. Social network theory: new insights and issues for behavioral ecologists. - Behav. Ecol. Sociobiol. 63: 975-988.

Smith, M. J. et al. 2009. Host-pathogen time series data in wildlife support a transmission function between density and frequency dependence. - Proc. Natl. Acad. Sci. 106: 7905-7909.

Stilling, P. D. 1988. Density-dependent processes and key-factors in insect populations. - J. Anim. Ecol. 57: 581-593.

Stoffel, M. et al. 2011. rptR: Repeatability for Gaussian and non-Gaussian data. in press.

Travis, S. et al. 1995. Ecological and demographic effects on intraspecific variation in the social system of Prairie dogs. - Ecology 76: 1794-1803.

Vander Wal, E. et al. 2012. Sex-based differences in density-dependent sociality: an experiment with a gregarious ungulate. - Ecology 93: 206-212.

Veenstra, A. J. F. 1958. The behaviour of the multimammate mouse, Rattus (Mastomys) natalensis (A. Smith). - Anim. Behav. 6: 195-206.

Warner, R. and Hoffman, S. 1980. Local population size as a determinant of mating system and sexual composition in two tropical marine fishes (Thalassoma spp.). - Evolution 34: 508-518.

Whitehead, H. 2008. Analyzing animal societies: quantitative methods for vertebrate social analysis. University of Chicago Press.

Woolhouse, M. E. J. 1997. Heterogeneities in the transmission of infectious agents: Implications for the design of control programs. - Proc. Natl. Acad. Sci. 94: 338-342. 
Table Legends

Table 1. Weekly number of individuals in the enclosures.

\begin{tabular}{ccccc}
\hline $\mathrm{A}+\mathrm{B}$ & 1 & 5 & 5 \\
$\mathrm{~A}+\mathrm{B}$ & 2 & 5 & 10 \\
$\mathrm{~A}+\mathrm{B}$ & 3 & 10 & 20 \\
$\mathrm{~A}+\mathrm{B}$ & 4 & 10 & 30 \\
$\mathrm{~A}+\mathrm{B}$ & 5 & 10 & 40 \\
$\mathrm{C}+\mathrm{D}$ & 1 & 60 & 60 \\
$\mathrm{C}+\mathrm{D}$ & 2 & 20 & 80 \\
$\mathrm{C}+\mathrm{D}$ & 3 & 20 & 100 \\
$\mathrm{C}+\mathrm{D}$ & 4 & 20 & 120 \\
$\mathrm{C}+\mathrm{D}$ & 5 & 16 & 136 \\
\hline
\end{tabular}

'This article is protected by copyright. All rights reserved.' 
Table 2. AIC and P values for the different model fits to the network parameter - density data.

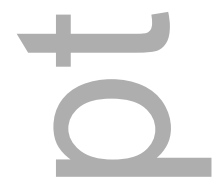

\begin{tabular}{|c|c|c|c|c|c|c|}
\hline & \multicolumn{3}{|c|}{ AIC } & \multicolumn{3}{|c|}{ P-value } \\
\hline & Linear & Power & Sigmoid & Linear-Power & Linear-Sigmoid & Power-Sigmoid \\
\hline Degree & 39.7 & 41.1 & 34.3 & 0.448 & 0.018 & 0.007 \\
\hline Betweenness & 271.4 & 271.8 & 272.1 & 0.223 & 0.219 & 0.213 \\
\hline Closeness & 209.3 & 211.1 & 211 & 0.635 & 0.349 & 0.173 \\
\hline
\end{tabular}
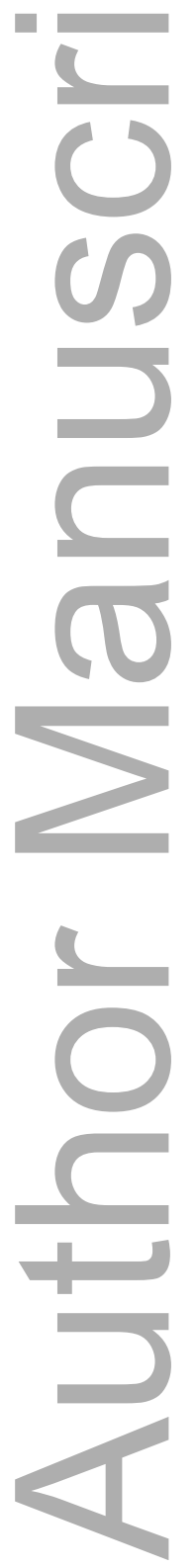

'This article is protected by copyright. All rights reserved.' 


\section{Figure Legends}

Figure 1. Mean degree $( \pm \mathrm{SE})$ for each density, with fitted contact-density functions. The $\mathrm{x}$-axis shows the number of animals per 0.5 ha enclosure. AIC values are shown in the legend.

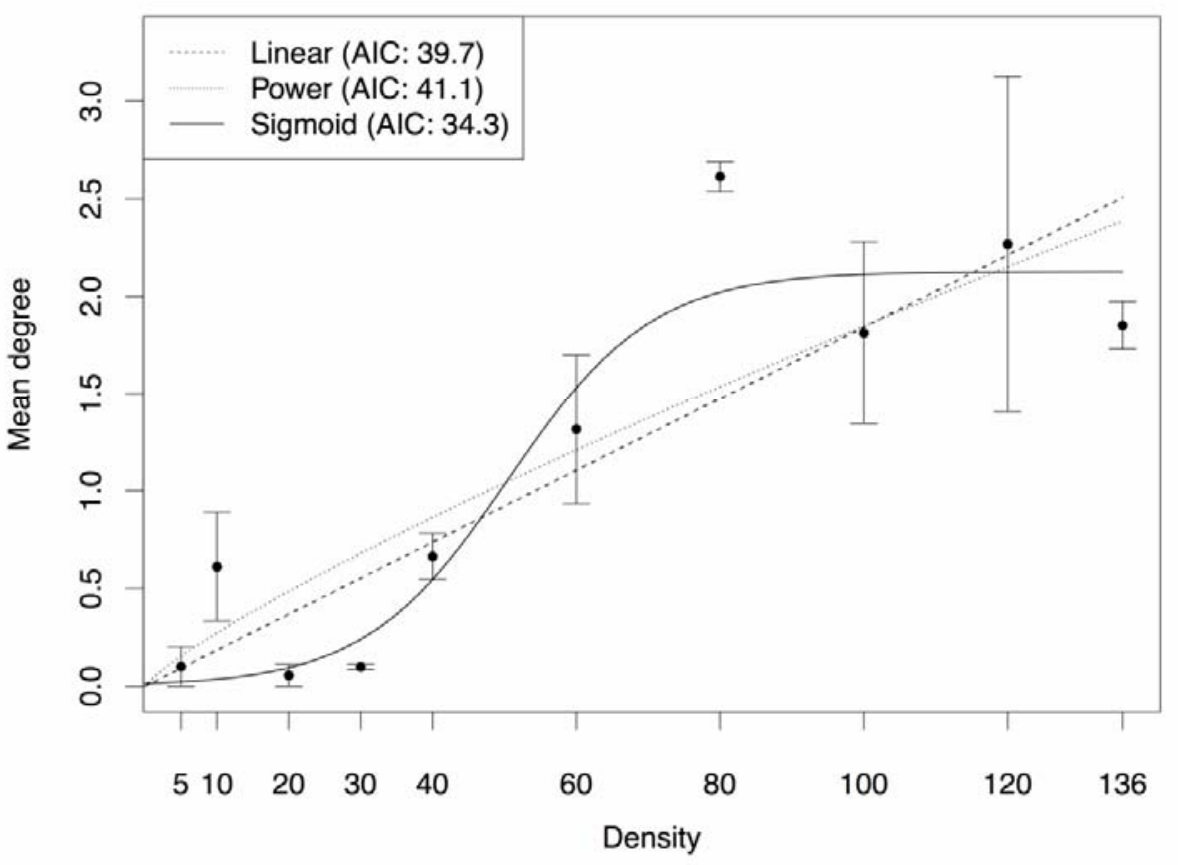

'This article is protected by copyright. All rights reserved.' 
Figure 2. Mean degree of the observed (crosses) and the randomised (grey dots) networks, for each enclosure-density combination. P-values $<0.05(* * *)$ indicate when the observed degree was significantly lower or higher than that of random networks.

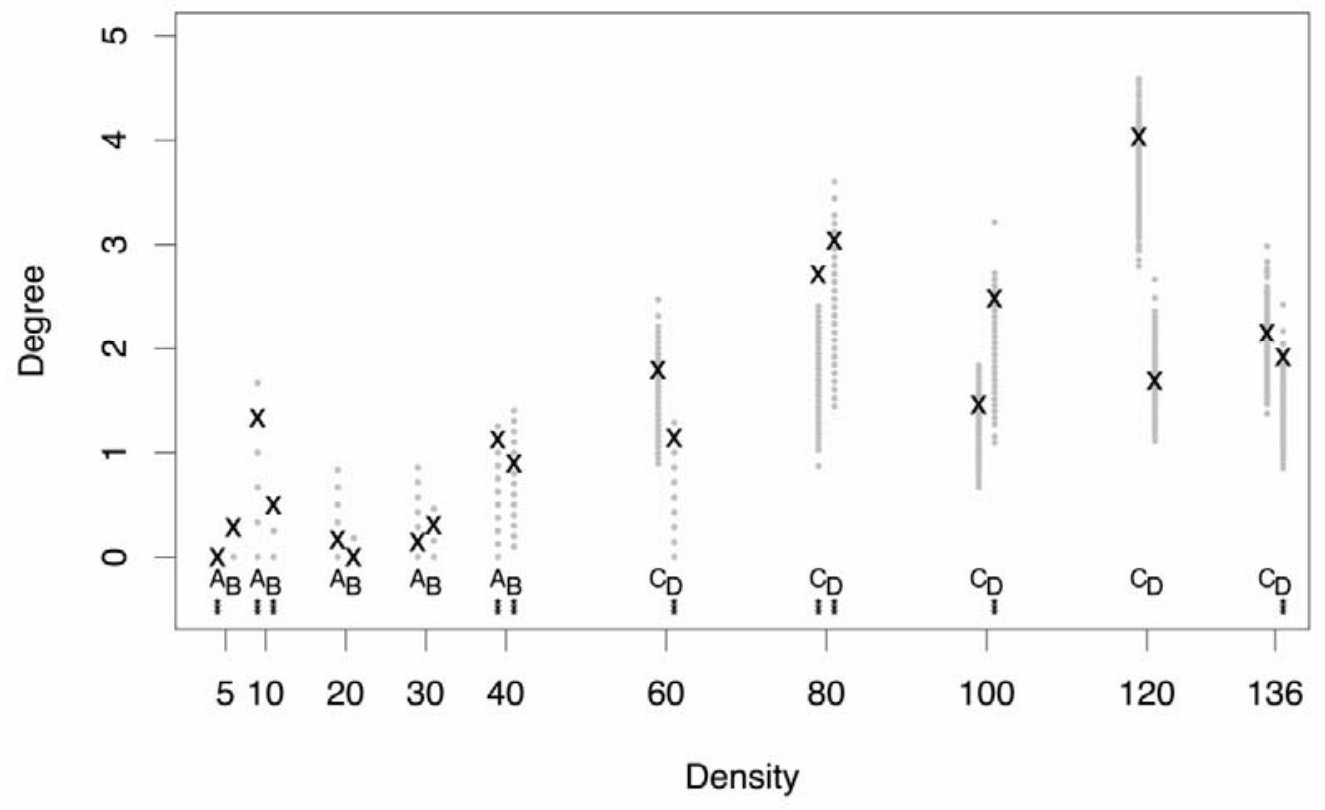

'This article is protected by copyright. All rights reserved.' 
Figure 3. Mean $( \pm \mathrm{SE})$ betweenness and closeness for each density. Betweenness is the number of shortest indirect connections between any two individuals in the network that pass through a focus individual. A closeness value of 0 occurs when an individual is unconnected to others, while higher closeness values indicate that the average distance of an individual to all other individuals is small. The xaxis shows the number of animals per 0.5 ha enclosure.

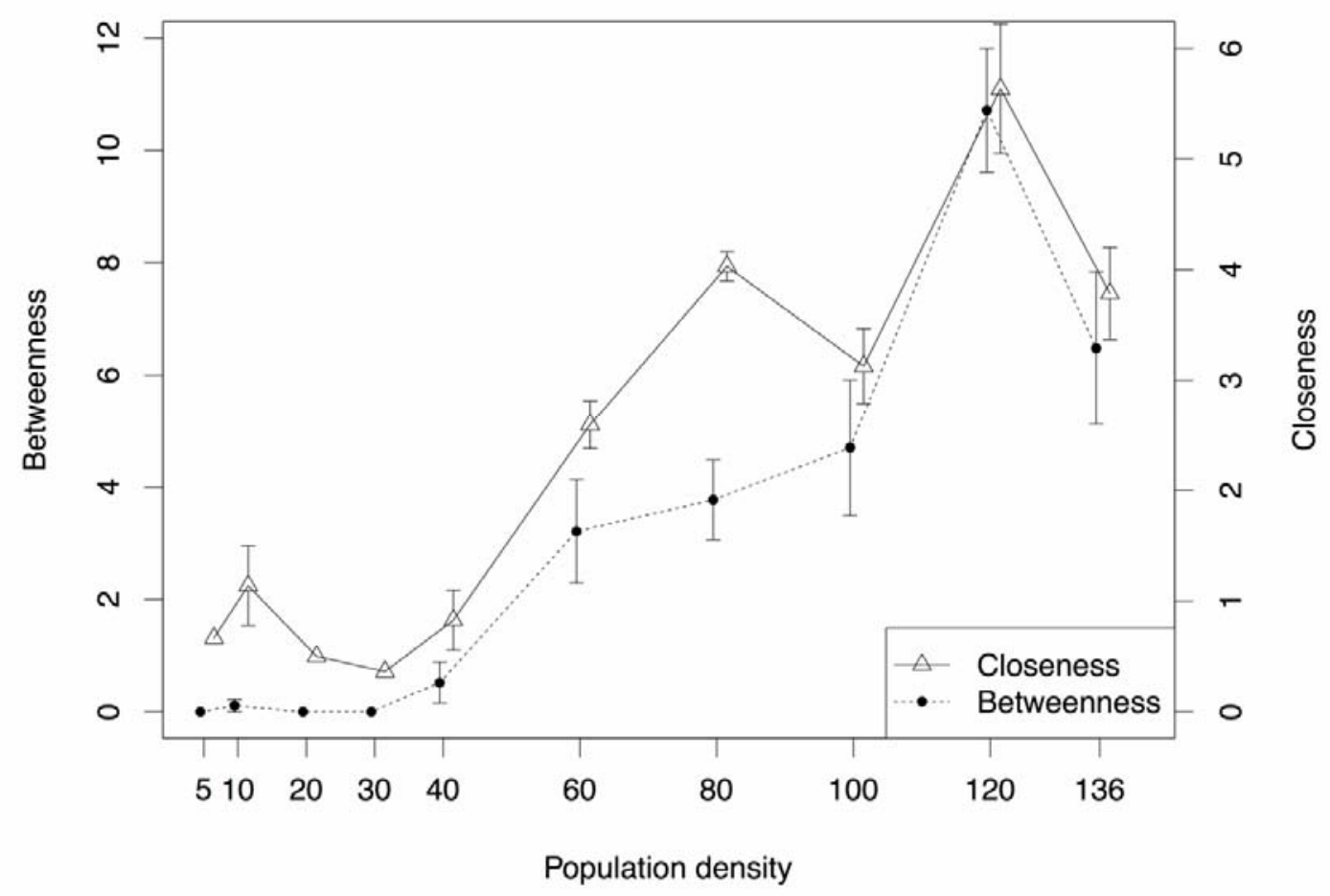

'This article is protected by copyright. All rights reserved.' 
Figure 4. Frequency distributions of normalized degree (left), betweenness (middle) and closeness (right) for each population density. Darker colours indicate a larger proportion of individuals within the network, with proportions ranging from 0 to 1 . Skewness is indicated by the dotted lines. Population density is the number of individuals in a 0.5 ha enclosure.
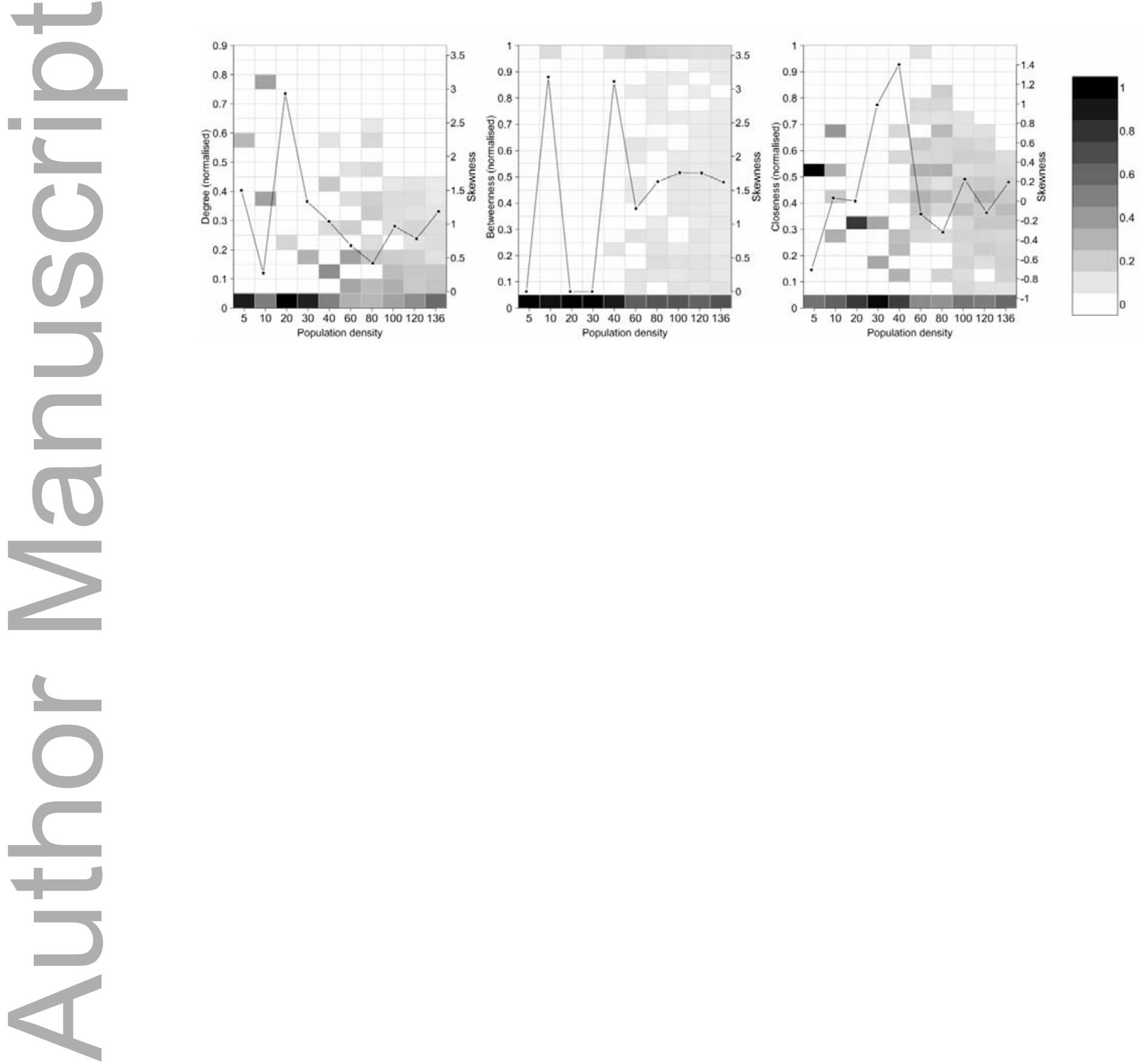

'This article is protected by copyright. All rights reserved.' 
Figure 5. Number of active minutes (left) and coefficient of variation (right) per individual (mean $\pm \mathrm{SE}$ ) vs. population density.
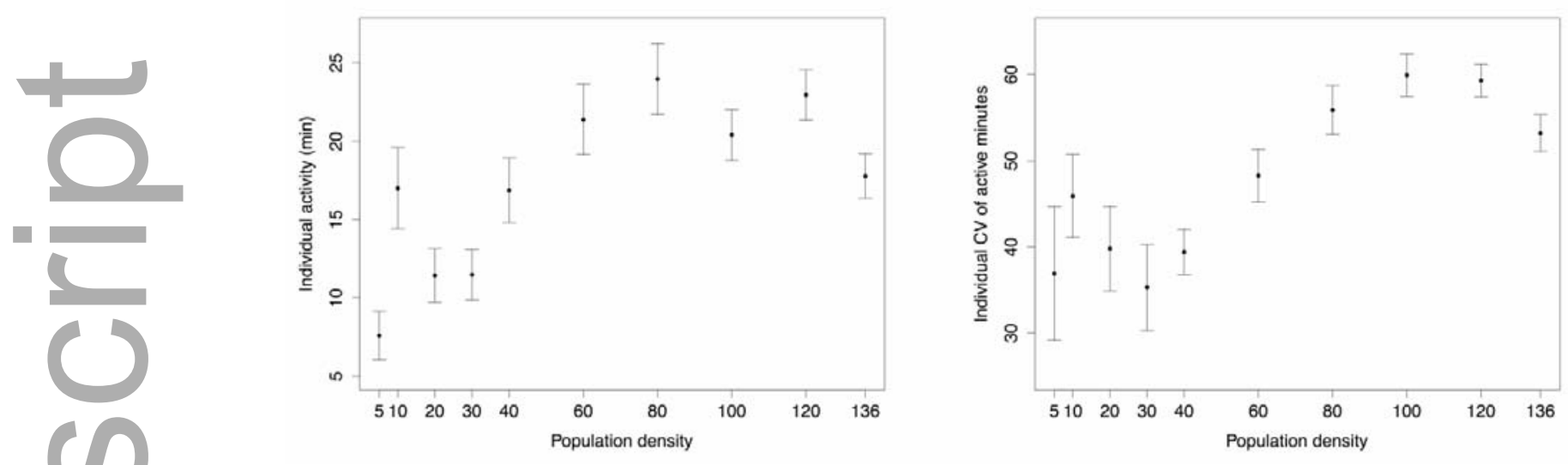

'This article is protected by copyright. All rights reserved.' 


\section{University Library}

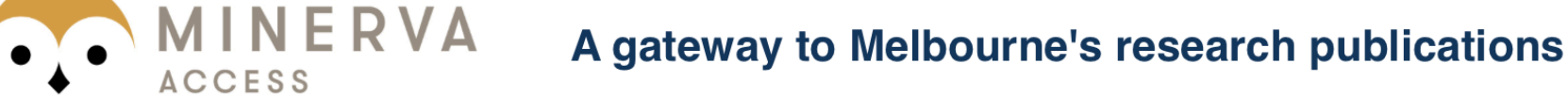

Minerva Access is the Institutional Repository of The University of Melbourne

Author/s:

Borremans, B;Reijniers, J;Hughes, NK;Godfrey, SS;Gryseels, S;Makundi, RH;Leirs, H

Title:

Nonlinear scaling of foraging contacts with rodent population density

Date:

2017-06-01

Citation:

Borremans, B., Reijniers, J., Hughes, N. K., Godfrey, S. S., Gryseels, S., Makundi, R. H.

\& Leirs, H. (2017). Nonlinear scaling of foraging contacts with rodent population density.

OIKOS, 126 (6), pp.792-800. https://doi.org/10.1111/oik.03623.

Persistent Link:

http://hdl.handle.net/11343/292109 